\title{
Dirty black holes: quasinormal modes for 'squeezed' horizons
}

\author{
A J M Medved, Damien Martin and Matt Visser \\ School of Mathematical and Computing Sciences, Victoria University of Wellington, \\ PO Box 600, Wellington, New Zealand \\ E-mail: joey.medved@mcs.vuw.ac.nz,damien.martin@mcs.vuw.ac.nz and \\ matt.visser@mcs.vuw.ac.nz \\ Received 29 October 2003 \\ Published 7 April 2004 \\ Online at stacks.iop.org/CQG/21/2393 (DOI: 10.1088/0264-9381/21/9/013)
}

\begin{abstract}
We consider the quasinormal modes for a class of black-hole spacetimes that, informally speaking, contain a closely 'squeezed' pair of horizons. (This scenario, where the relevant observer is presumed to be 'trapped' between the horizons, is operationally distinct from near-extremal black holes with an external observer.) It is shown, by analytical means, that the spacing of the quasinormal frequencies equals the surface gravity at the squeezed horizons. Moreover, we can calculate the real part of these frequencies provided that the horizons are sufficiently close together (but not necessarily degenerate). The novelty of our analysis (which extends a model-specific treatment by Cardoso and Lemos) is that we consider 'dirty' black holes, that is, the observable portion of the (static and spherically symmetric) spacetime is allowed to contain an arbitrary distribution of matter.
\end{abstract}

PACS numbers: 04.70.-s, 04.30.-w

\section{Introduction}

The perturbations of the spacetime outside of a black-hole horizon are expected to be radiated away, at late times, with a discrete set of complex-valued frequencies, the so-called quasinormal mode frequencies of a black hole [1-3]. Knowledge of these modes should have particular importance in gravitational-wave astronomy and, in a more speculative scenario, may even provide insight into the very essence of black-hole entropy (which still lacks a convincing statistical explanation).

With regard to the latter motivation, an interesting proposal has been put forth by Hod [4]. On the basis of Bohr's correspondence principle, Hod has suggested that, in the asymptotic 
limit of a 'highly damped' black hole ${ }^{1}$, the real part of the quasinormal frequency should represent a characteristic (transition) frequency for the black hole itself. Moreover, it was then argued that the value of this special frequency could be used as a means for uniquely fixing the level spacing of the black-hole area spectrum. (The concept of a uniformly spaced area spectrum for black holes was first advocated by Bekenstein [5].)

To help flesh out these somewhat esoteric statements, let us consider the asymptotic behaviour of the quasinormal modes for a Schwarzschild black hole. In the case of scalar or gravitational perturbations, these asymptotic frequencies are known to take the following form $[6,7]$ :

$$
k_{q n m}(n)=\frac{1}{4 m}\left[\mathrm{i}\left(n+\frac{1}{2}\right)+\frac{\ln 3}{2 \pi}\right]+\mathcal{O}\left[n^{-1 / 2}\right] \quad \text { as } \quad n \rightarrow \infty,
$$

where $n$ is the quantum number that labels the frequencies and $m$ is the black-hole mass ${ }^{2}$. Note that the asymptotic spacing, or the 'gap', is just the Schwarzschild surface gravity $\left(\kappa_{s}=1 / 4 m\right)$. According to Hod [4], one can identify the real part of the subleading term, or the 'offset', as the 'transition frequency' $\omega$ so that $\delta m=\omega=\kappa_{s} \ln 3 / 2 \pi$. Then, given the Schwarzschild area formula or $A=16 \pi \mathrm{m}^{2}$, it immediately follows that $\delta A=32 \pi m \delta m=4 \ln 3$ equals the spacing between adjacent levels in the area spectrum. It is an interesting consequence that, with this particular spacing, $\exp (S)$ takes on an integer value (where $S=A / 4$ is the black-hole entropy) in compliance with statistical expectations.

The above considerations have helped trigger a recent surge of research activity in the realm of quasinormal modes. For instance, there has been some discussion on linking the intriguing appearance of $\ln 3$ with the gauge group of loop quantum gravity (e.g. [8, 9]). Furthermore, there has also been a considerable amount of work on evaluating the quasinormal frequencies for various black-hole models, both numerically (e.g. [10, 11]) and analytically (e.g. [12-14]). For a more thorough list of references, see [15, 16].

It is worth pointing out that most of these frequency calculations have been highly model specific. (For a recent status report and summary, see [16].) On the other hand, it is the opinion of the present authors that significant progress will almost certainly require more general considerations. This perspective has prompted a recent study [15] in which we investigated the asymptotic mode behaviour for a very general class of spacetimes: that of 'dirty' black holes. These are static, spherically symmetric but otherwise generic spacetimes for which there is a central black hole surrounded by arbitrary matter fields [17-19]. (Keep in mind that such geometries also allow for multi-horizon scenarios and the presence of a cosmological horizon.)

Although our prior treatment [15] confirmed that the gap going as the surface gravity is a generic result, it was unable to say anything about the real part of the offset. In this paper, however, we are able to rectify this omission for a certain class of dirty black-hole spacetimes. More specifically, we will now focus on a scenario of 'squeezed' horizons. That is to say, the observable part of the spacetime is bounded by a pair of horizons which are, in a well-defined sense, regarded as being 'close'. (This should not be confused with the case of a nearly extremal black hole where the observer is, most typically, regarded as residing in the exterior portion of the spacetime.) For this class of spacetimes, it will be shown that the offset can, in fact, be calculated exactly in the squeezing limit. This will be accomplished by casting the (generic) 'scattering potential' into a recognizable, Poschl-Teller form [21]. Notably, the same basic technique has been used by Cardoso and Lemos [22] for the specific case of a

1 By highly damped, it is meant that the imaginary portion of the mode frequency has become very large. This connection follows from the imaginary part being a measure of the inverse relaxation time of a radiating black hole.

2 Here and throughout, all fundamental constants are fixed to unity and a four-dimensional spacetime is presumed. 
Schwarzschild-de Sitter ('squeezed') geometry; nevertheless, we are now able to demonstrate that their results have much more general applicability.

The remainder of the paper is arranged as follows. In the following section, we present the basic formalism and set up conventions. The third section contains the main analysis, that is, the calculation of the quasinormal mode frequencies as previously discussed. The main body of the paper ends with a brief summary and discussion. There is also an appendix where the scattering potential is rigorously derived for axial gravitational perturbations in a dirty black-hole background (the scalar-perturbation calculation is documented in [15]).

\section{The formal preliminaries}

Let us begin by introducing our so-called dirty black-hole spacetime. That is, a spherically symmetric and static_-but otherwise general-black-hole geometry (alternatively, the geometry of a central black hole surrounded by arbitrary matter fields). Without loss of generality, the metric for such a spacetime can always be expressed as (e.g., [17, 23])

$$
\mathrm{d} s^{2}=-\mathrm{e}^{-2 \phi(r)}\left[1-\frac{2 m(r)}{r}\right] \mathrm{d} t^{2}+\left[1-\frac{2 m(r)}{r}\right]^{-1} \mathrm{~d} r^{2}+r^{2} \mathrm{~d} \Omega^{2},
$$

where $\phi(r)$ and $m(r)$ are model-dependent functions (related to the Morris-Thorne 'redshift' and 'shape' functions, respectively [24]). Keep in mind that $m(r)$ retains its usual interpretation as a mass parameter, whereas $\phi(r)$ measures any deviation (due to matter fields) from the 'Schwarzschild form' of $g_{r r}^{-1}=-g_{t t}$.

It often proves useful to have the metric expressed in terms of a generalized tortoise coordinate,

$$
\frac{\mathrm{d} r_{*}}{\mathrm{~d} r}=\frac{\mathrm{e}^{\phi(r)}}{1-\frac{2 m(r)}{r}}
$$

so that the line element (2) can now be written as

$$
\mathrm{d} s^{2}=\mathrm{e}^{-2 \phi(r)}\left(1-\frac{2 m(r)}{r}\right)\left[-\mathrm{d} t^{2}+\mathrm{d} r_{*}^{2}\right]+r^{2} \mathrm{~d} \Omega^{2} .
$$

Note that our formalism does, in its full generality, allow for spacetimes with more than one causal horizon, one of which may be a cosmological horizon (if the spacetime is asymptotically de Sitter). For any such horizon - that is, any radius $r=r_{h}$ which satisfies $r_{h}=2 m\left(r_{h}\right)$-one can calculate the surface gravity via standard methods [17]. More specifically, for a black-hole (event) horizon,

$$
\begin{aligned}
\kappa_{h} & =\left.\frac{1}{2} \frac{\mathrm{d}}{\mathrm{d} r}\left[\mathrm{e}^{-\phi(r)}\left(1-\frac{2 m(r)}{r}\right)\right]\right|_{r_{h}} \\
& =\frac{1}{2 r_{h}} \mathrm{e}^{-\phi\left(r_{h}\right)}\left[1-8 \pi \rho\left(r_{h}\right) r_{h}^{2}\right],
\end{aligned}
$$

where $\rho(r)$ is the energy density. In contrast, one would take the negative of the above expression to obtain the surface gravity at a cosmological horizon [25]. In this case, the non-negativity of surface gravity immediately implies a lower bound on the radius of any cosmological horizon, $r_{c}$, as a function of the energy density, namely, $r_{c}^{2} \geqslant\left[8 \pi \rho\left(r_{c}\right)\right]^{-1}$. This inequality can be viewed as a generalization of the Nariai bound [26], that is, the upper limit on the mass of a black hole in a Schwarzschild-de Sitter [Kottler] geometry.

Let us now assume the existence of two non-degenerate horizons, say, $r_{a}$ and $r_{b}$ with $r_{b}>r_{a}$ and no other horizons inbetween. (There can, however, still be other horizons 
in the spacetime, but these would be operationally irrelevant to our treatment - see below.) When considering this particular scenario (especially relevant to section 3), we will always view matters from the perspective of an observer who is 'trapped' between the two horizons. Hence, considerations can safely be restricted to the submanifold defined by $r_{a} \leqslant r \leqslant r_{b}$. (It is useful to remember that, for this setup, the region $r \in\left(r_{a}, r_{b}\right)$ maps onto the region $r_{*} \in(-\infty,+\infty)$.) Under these circumstances, the metric components of interest can always be expressed as

$$
g_{r r}^{-1}=1-\frac{2 m(r)}{r}=\frac{\left(r-r_{a}\right)\left(r_{b}-r\right)}{r^{2}} h(r)
$$

and

$$
\left|g_{t t}\right|=\mathrm{e}^{-2 \phi(r)}\left(1-\frac{2 m(r)}{r}\right)=\mathrm{e}^{-2 \phi(r)} \frac{\left(r-r_{a}\right)\left(r_{b}-r\right)}{r^{2}} h(r),
$$

where $h(r)$ is some dimensionless function that is well behaved, regular and positive throughout the observable portion of the manifold. Note that one can now write the mass as $2 m(r) / r=1-\left(r-r_{a}\right)\left(r_{b}-r\right) h(r) / r^{2}$, indicating that $h(r)$ can be physically interpreted as (a dimensionless measure of ) the energy density of the extraneous or 'dirty' matter.

For future convenience, let us re-express some earlier formalism directly in terms of the function $h(r)$ or, rather, the modified form (for calculational simplicity) $H(r) \equiv \mathrm{e}^{-\phi(r)} h(r) / r^{2}$. The defining expression for the generalized tortoise coordinate (3) now becomes

$$
\frac{\mathrm{d} r_{*}}{\mathrm{~d} r}=\left[\left(r-r_{a}\right)\left(r_{b}-r\right) H(r)\right]^{-1},
$$

whereas the surface gravity (5) can be re-evaluated to give (for the inner and outer horizon, respectively)

$$
\begin{aligned}
\kappa_{a} & =\frac{1}{2}\left(r_{b}-r_{a}\right) H\left(r_{a}\right), \\
\kappa_{b} & =\frac{1}{2}\left(r_{b}-r_{a}\right) H\left(r_{b}\right) .
\end{aligned}
$$

Since the primary interest of this paper is the calculation of quasinormal mode frequencies, let us remind the reader of some pertinent points. One considers small perturbations of the spacetime outside of the relevant horizon(s). As is well known in the Schwarzschild case, such considerations ultimately lead to a one-dimensional Schrödinger-like equation [27], that is,

$$
\frac{\mathrm{d}^{2}}{\mathrm{~d} r_{*}^{2}} \psi-V\left[r\left(r_{*}\right)\right] \psi=-k^{2} \psi,
$$

where $\psi=\psi\left[r\left(r_{*}\right)\right]$ describes the radial behaviour of the (massless) perturbation field, $k$ is the frequency and, for a scalar ( $\operatorname{spin} j=0$ ) field in particular, the 'scattering potential' is found to be

$$
V(r)=\left(1-\frac{2 m}{r}\right)\left[\frac{\ell(\ell+1)}{r^{2}}+\frac{2 m}{r^{3}}\right]
$$

with $\ell$ representing the orbital angular momentum $(\ell=0,1,2, \ldots)$. In the case of higher-spin $(j>0)$ fields, there is an often quoted generalization of this result [28] whereby one makes the replacement $2 m \rightarrow 2 m\left(1-j^{2}\right)$ in the numerator of the right-most term. However, it is certain that this generalization cannot in all circumstances be correct. See the appendix for further discussion.

We have, quite recently [15], generalized the scalar-perturbation potential to the generic spacetime described by equations (2) and (4). The one-dimensional Schrödinger-like form (11) 
does indeed persist, although the scattering potential becomes somewhat more complicated. More precisely,

$V(r)=\mathrm{e}^{-2 \phi(r)}\left(1-\frac{2 m(r)}{r}\right)\left[\frac{\ell(\ell+1)}{r^{2}}+2 \frac{m(r)}{r^{3}}-2 \frac{m^{\prime}(r)}{r^{2}}-\left(1-\frac{2 m(r)}{r}\right) \frac{\phi^{\prime}(r)}{r}\right]$,

where a prime denotes a derivative with respect to $r$. It is unclear if there is a simple way to generalize this result to arbitrary values of spin, $j$. For instance, the form of the potential for the $j=2$ case of axial gravitational perturbations (which we derive in the appendix) could not have easily been anticipated. (That is, one could not just make the type of naive replacement that was discussed above.) Nonetheless, we propose that the precise dependence of the potential on $j$ is, qualitatively speaking, inconsequential to the prior [15] or the current treatment.

The quasinormal modes can be regarded as the complex frequency solutions of equation (11) when 'radiation boundary conditions' are imposed at the boundaries of the observable spacetime [1,3]. (Complex values of $k$ are, in fact, necessitated by having a 'mostly positive' potential.) With regard to the case of a Schwarzschild black hole, the quasinormal mode frequencies are known to be labelled by a discrete quantum number [2] and have a well-known asymptotic form, exhibited in equation (1) and amply confirmed both numerically $[6,7]$ and analytically $[12,13]$.

More generally, we have recently shown that, up to the leading asymptotic order, the Schwarzschild form for the mode frequencies is, indeed, a generic feature of black-hole spacetimes [15]. More to the point, the imaginary spacing between the modes (or the 'gap') is, in the asymptotic limit, exactly equal to the relevant surface gravity under quite generic circumstances. Also in the cited work, we have extended considerations to an observer who is trapped between two horizons (precisely, the scenario discussed earlier in this section) and argued for the following generalization ${ }^{3}$ :

$$
k_{q n m}(n)=\mathrm{i}\left[n_{a} \kappa_{a}+n_{b} \kappa_{b}\right]+O[1] \quad \text { when } \quad n_{a}, n_{b} \rightarrow \infty
$$

Let us re-emphasize, however, that our prior approach [15]—which utilized the first Born approximation to the scattering amplitude-could not say anything about the real part of the order-unity term or the 'offset'.

\section{The main analysis}

We will now proceed to show that, for a certain class of observers, an exact calculation of the offset is still 'generically' possible ${ }^{4}$. To elaborate, we will return to our scenario of an observer trapped between horizons at $r_{a}$ and $r_{b}$, and then consider the 'squeezed'-horizon limit of $r_{a} \rightarrow r_{b}$ (or vice versa). (This is, of course, just the degenerate or extremal horizon limit; nonetheless, we have avoided using such terminology, as the usual inference is an observer exterior to both horizons (e.g., an observer outside of a Reissner-Nordström black hole.) Note that the following analysis generalizes a prior work, specific to the case of a Schwarzschild-de Sitter spacetime, by Cardoso and Lemos [22].

Let us begin here by establishing what is exactly meant by a spacetime with squeezed horizons. For the duration, the horizons will be regarded as sufficiently close so that

\footnotetext{
3 The same expression has also been deduced, by independent means, for the specific model of a Schwarzschild-de Sitter black hole [29]. However, for a contrary opinion, see [20].

4 By generic, we mean that the spacetime can be arbitrarily dirty.
} 
$\Delta \equiv\left(r_{b}-r_{a}\right) / r_{a} \ll 1$. Given the extent of the relevant manifold $\left(r_{a} \leqslant r \leqslant r_{b}\right)$, it immediately follows that, up to corrections of the order $\Delta, r_{a} \sim r \sim r_{b}$.

In view of the above, it is clear that much of the prior formalism will simplify. For instance, let us recall equation (8) for the tortoise coordinate. Up to corrections of the relative order $\Delta$ (always signified by $\sim$ ), we can write

$$
\frac{\mathrm{d} r_{*}}{\mathrm{~d} r} \sim\left[\left(r-r_{a}\right)\left(r_{b}-r\right) H\left(r_{a}\right)\right]^{-1} .
$$

To justify this, recall that $H(r)=\mathrm{e}^{-\phi(r)} h(r) / r^{2}$ is a regular, well-defined and positive function throughout the observable region (cf, equations (6) and (7)). We can then safely approximate

$$
H\left(r_{a}\right) \sim H(r) \sim H\left(r_{b}\right)
$$

in the region of interest $r \in\left(r_{a}, r_{b}\right)$. In particular,

$$
\kappa_{a} \sim \kappa_{b}
$$

up to corrections of higher order in $\Delta$. The approximate relation for the tortoise coordinate can now readily be integrated to give

$$
r_{*} \sim \frac{1}{\left(r_{b}-r_{a}\right) H\left(r_{a}\right)} \ln \left[\frac{r-r_{a}}{r_{b}-r}\right]=\frac{1}{2 \kappa_{a}} \ln \left[\frac{r-r_{a}}{r_{b}-r}\right],
$$

which can then be inverted to yield

$$
r \sim \frac{r_{a}+r_{b} \exp \left[2 \kappa_{a} r_{*}\right]}{1+\exp \left[2 \kappa_{a} r_{*}\right]}=\frac{r_{a}+r_{b}}{2}+\frac{r_{b}-r_{a}}{2} \tanh \left[\kappa_{a} r_{*}\right]
$$

Applying equation (9), we can directly substitute the above relation for $r=r\left(r_{*}\right)$ into $\left(r-r_{a}\right)\left(r_{b}-r\right) H\left(r_{a}\right)$ and obtain (after some manipulations)

$$
\left(r-r_{a}\right)\left(r_{b}-r\right) H\left(r_{a}\right) \sim \frac{\kappa_{a}\left(r_{b}-r_{a}\right)}{2 \cosh ^{2}\left[\kappa_{a} r_{*}\right]} .
$$

But, given that $H(r) \sim H\left(r_{a}\right)$ throughout the relevant manifold, this also means that

$$
\mathrm{e}^{-\phi(r)}\left(1-\frac{2 m(r)}{r}\right)=\left(r-r_{a}\right)\left(r_{b}-r\right) H(r) \sim \frac{\kappa_{a}\left(r_{b}-r_{a}\right)}{2 \cosh ^{2}\left[\kappa_{a} r_{*}\right]} .
$$

It is useful to note that the left-hand side is essentially the time-time component of the metric (cf, equation (7)), up to a 'distortion' which can be regarded as a constant factor in the regime of interest.

The above result is, basically, all that is needed to ascertain the quasinormal modes. To demonstrate this, we will initially concentrate on the simplest case of a scalar perturbation and then comment on general values of $j$ below. To begin here, let us first call upon the generic form of the scattering potential for scalar fields (13). Conveniently, one can always re-express this potential in the following compact form [15]:

$$
V(r)=\mathrm{e}^{-2 \phi(r)}\left(1-\frac{2 m(r)}{r}\right) \frac{\ell(\ell+1)}{r^{2}}+\frac{1}{r}\left(\partial_{r_{*}}^{2} r\right) .
$$

Evaluating the partial derivatives with the help of equation (8), we find that

$$
\begin{aligned}
V(r)=\left(r-r_{a}\right) & \left(r_{b}-r\right) H(r) \\
& \times\left[\mathrm{e}^{-\phi(r)} \frac{\ell(\ell+1)}{r^{2}}+\frac{\left(r-r_{a}\right)\left(r_{b}-r\right) H^{\prime}(r)}{r}+\frac{\left(r_{a}+r_{b}-2 r\right) H(r)}{r}\right] .
\end{aligned}
$$

So far, everything is exact; however, applying the 'squeezed approximation' $(\Delta \ll 1)$ and equation (21) in particular, we then get

$$
V\left(r_{*}\right) \sim \mathrm{e}^{-\phi\left(r_{a}\right)} \frac{\kappa_{a}\left(r_{b}-r_{a}\right)}{2 \cosh ^{2}\left[\kappa_{a} r_{*}\right]} \frac{\ell(\ell+1)}{r_{a}^{2}}=\frac{\kappa_{a}^{2}}{h\left(r_{a}\right)} \frac{\ell(\ell+1)}{\cosh ^{2}\left[\kappa_{a} r_{*}\right]} .
$$


To put it another way,

$$
V\left(r_{*}\right) \sim \frac{V_{j=0, \ell}}{\cosh ^{2}\left[\kappa_{a} r_{*}\right]},
$$

where $V_{0, \ell}$ is a model-dependent quantity (in principle, always calculable) that is constant for any fixed value of $\ell$.

Significantly, equation (25) can be identified as the Poschl-Teller potential [21]: a form of potential for which the one-dimensional scattering equation (11) can readily be solved. Choosing appropriate plane-wave boundary conditions for the perturbation field, $\Psi \sim \exp \left[\mathrm{i} k\left(t \pm r_{*}\right)\right]$ as $r_{*} \rightarrow \mp \infty$, one finds that [30]

$$
k_{q n m} \sim \kappa_{a}\left[\mathrm{i}\left(n+\frac{1}{2}\right)+\sqrt{\frac{V_{0, \ell}}{\kappa_{a}^{2}}-\frac{1}{4}}\right] \quad \text { where } n=0,1,2, \ldots
$$

This result generalizes equation (20) in [22], which was specific to the case of a ('squeezed') Schwarzschild-de Sitter black hole. It is easily confirmed that our form of the potential (24) reduces to theirs for this special model.

Take note of the gap or spacing between the levels at large $n$; this is precisely $\kappa_{a} \sim \kappa_{b}$, an outcome which agrees with our prior findings (cf, equation (14)). Moreover, the offset can now be trivially extracted:

$$
\mathrm{i} \frac{\kappa_{a}}{2}+\sqrt{V_{0, \ell}-\frac{\kappa_{a}^{2}}{4}}=\mathrm{i} \frac{\kappa_{a}}{2}+\kappa_{a} \sqrt{\frac{\ell(\ell+1)}{h\left(r_{a}\right)}-\frac{1}{4}} .
$$

Besides the surface gravity, the frequencies depend on only one other parameter of the spacetime, this being the dimensionless parameter $h\left(r_{a}\right)$. On the basis of dimensional arguments, one might expect $h\left(r_{a}\right)$ to be, at the very most, of the order unity and is, in fact, exactly unity for the special case of Schwarzschild-de Sitter space. (It is also worth pointing out that $h(r)$ depends on the mass parameter, $m(r)$, but not on the redshift function, $\phi(r)$, that is, the quasinormal modes, even at the level of the offset, cannot give us a very detailed account of the dirty matter.) Therefore, one can see that, for any $\ell>0$, the argument of the square root is positive and roughly proportional to $\ell^{2}$. (Curiously, such explicit $\ell$ dependence is notably absent in many studies on the quasinormal modes for an exterior observer (e.g., $[6,7,12,13])$. Nonetheless, there is no obvious contradiction here, as no such work caters to our squeezed-horizon scenario.)

Meanwhile, $\ell=0$ requires special consideration, since the square-bracket quantity in equation (23) for the potential will then vanish up to the order of $\Delta$. If one goes on to examine the next-order term in the brackets, it becomes evident that the potential can no longer be cast into the Poschl-Teller form. Nevertheless, it can be argued that this extra factor of $\Delta$ (for $\ell=0$ the potential is of order $\Delta^{3}$ instead of $\Delta^{2}$ ) causes the potential to become negligible.

It should be emphasized that, although approximations have been used in attaining the Poschl-Teller form, equation (26) provides an exact description of the quasinormal mode behaviour in the squeezing limit. This is because any neglected terms in the potential are of, at most, the relative order $\Delta$ and $\Delta=\left(r_{b}-r_{a}\right) / r_{a} \propto \kappa_{a}$. Hence, we can more precisely write

$$
k_{q n m} \rightarrow \kappa_{a}\left[\mathrm{i}\left(n+\frac{1}{2}\right)+\sqrt{\frac{\ell(\ell+1)}{h\left(r_{a}\right)}-\frac{1}{4}}\right] \quad \text { as } \quad \Delta \rightarrow 0 .
$$

Moreover, the validity of this result does not depend on the horizons becoming perfectly degenerate, they must only be sufficiently close so that the neglected terms in $V\left(r_{*}\right)$ (which are suppressed by at least $\mathcal{O}[\Delta]$ ) make a negligible contribution to the quasinormal mode 
spectrum. Indeed, there must be some range of nearly degenerate solutions for which this will be the case.

Before concluding, let us briefly comment on the case of general $j$. If one considers axial gravitational $(j=2)$ perturbations, then the scattering potential takes the form, as derived in the appendix, of equation (A.21). Closely following the prior methodology, we find that, in the squeezed-horizon limit, this potential now becomes

$$
V\left(r_{*}\right) \sim \frac{V_{2, \ell}}{\cosh ^{2}\left[\kappa_{a} r_{*}\right]}=\mathrm{e}^{-\phi\left(r_{a}\right)} \frac{\kappa_{a}\left(r_{b}-r_{a}\right)}{2 \cosh ^{2}\left[\kappa_{a} r_{*}\right]} \frac{[\ell(\ell+1)-2]}{r_{a}^{2}} .
$$

Hence, all the above outcomes carry through with the simple replacement $V_{0, \ell} \rightarrow V_{2, \ell}$, that is, $\ell(\ell+1) \rightarrow \ell(\ell+1)-2$. Although it is difficult to be precise about general values of spin, one might be tempted to suggest a 'generalization' of the form $\ell(\ell+1) \rightarrow \ell(\ell+1)+F[j]$, where $F[j]$ is some simple polynomial of leading order $j^{2}$. Nevertheless, even though the $j$ dependence probably deviates from such naive expectations, it is still clear that:

- the qualitative features of the analysis will persist, and

- for large enough values of $\ell$ the dependence on $j$ will become negligible.

\section{Conclusion}

In summary, we have determined the asymptotic frequencies of the quasinormal modes as would be measured by an observer who is trapped between a pair of 'squeezed' horizons. Although these circumstances are arguably special, our analysis is quite general in the sense that the spacetime can contain arbitrary matter fields or 'dirt'. That is, we have allowed for the most general horizon geometries in a static and spherically symmetric spacetime, thus extending the Schwarzschild-de Sitter treatment of Cardoso and Lemos [22].

We have found that the 'gap' (or asymptotic level spacing) coincides precisely with the surface gravity, $\kappa_{h}$ (at either horizon), in notable agreement with our other recent paper [15]. Moreover, the 'offset' (or subleading term) was found to go, roughly, as $\kappa_{h} \ell$ for large enough $\ell$ and $n$. Let us re-emphasize that our results are exact in the squeezing limit, that is, provided that the spacing between the horizons is small in units of horizon radius.

We should also stress that the scenario considered here is distinct from what is usually referred to, in the literature, as near extremality or horizon degeneracy. Studies on (nearly) extremal black holes usually presuppose an observer who remains exterior to both of the horizons. One should not expect the outcomes of this paper to translate into this other type of scenario. Indeed, in our related work [15], we have argued that such an exterior observer would likely find the quasinormal modes to collapse, asymptotically, to zero momentum ${ }^{5}$.

Finally, let us ponder upon the meaning (if any) of an offset term that depends so strongly on the orbital angular momentum, $\ell$, of the perturbation field. To remind the reader, the offset of a (for instance) Schwarzschild black-hole is believed to asymptote towards the same fixed value $[\kappa \ln (3) / 2 \pi]$ for any (fixed) choice of $\ell[6,7,12,13]$. There is, of course, no contradiction here; any scenario with an exterior observer is decidedly different from ours. Nonetheless, this 'discrepancy' does raise issues with a popular conjecture: the asymptotic value of the offset can be employed to fix the spacing between black-hole area eigenvalues $[4,8]$. If this were truly the case, then it seems rather strange that the asymptotic value of the offset can, at times, depend on the angular momentum of the particles being emitted. That is to say, one would expect this $\ell$ independence to persist for all types of black holes or, alternatively, why should only certain classes of horizons be subject to quantization? It would

\footnotetext{
5 Actually, the extremal situation is somewhat more complicated than this. See [15] for a detailed discussion.
} 
seem that the status of using quasinormal modes, in this particular context, requires further investigation.

\section{Acknowledgments}

The research was supported by the Marsden Fund administered by the New Zealand Royal Society and by the University Research Fund of Victoria University.

\section{Appendix. Wave equation for gravitational perturbations}

One can always see how a gravitational field is perturbed by simply perturbing the relevant metric. Holding the stress tensor fixed and studying the linear perturbations, one should then be able to extract the gravitational-wave equation and, thus, the scattering potential for a graviton. Note, however, that this technique says nothing about general $j=2$ fields, that is, we will be strictly considering $j=2$ perturbations due to gravity. (In this regard, let us point out that much of the quasinormal literature assumes generalizations (from the scalar case) that are straightforward, but (as observed by Cvetič and Larsen, and by Kanti and March-Russell [31]) such a naive approach fails to incorporate the Bianchi identities except in the vacuum equations, which is to say, the wave equation should, generally, depend on the type of field being discussed and not just its spin.) The computation reported below is an independent consistency check on that of Karlovini [32].

Allowing for the possibility of gravitational radiation, we will (closely following Chandrasekhar [33]) consider small perturbations of the metric and characterize these by $q_{r}, q_{t}$ and $q_{\theta}$ :

$$
\begin{aligned}
\mathrm{d} s^{2}=-\mathrm{e}^{-2 \phi(r)} & \left(1-\frac{2 m(r)}{r}\right) \mathrm{d} t^{2}+\left(1-\frac{2 m(r)}{r}\right)^{-1} \mathrm{~d} r^{2}+r^{2} \mathrm{~d} \theta^{2} \\
& +r^{2} \sin ^{2} \theta\left[\mathrm{d} \varphi-q_{r}(r, \theta, t) \mathrm{d} r-q_{\theta}(r, \theta, t) \mathrm{d} \theta-q_{t}(r, \theta, t) \mathrm{d} t\right]^{2} .
\end{aligned}
$$

(So far, all the functional dependence is explicit in the metric but, for the sake of brevity, this will not always be so in the following.) The above equation describes the so-called axial perturbations, for which a change in the sign of $\varphi$ necessitates that the perturbations must also change sign (for the metric to remain unchanged). It should be emphasized that these perturbations are not completely general; one could, just as well, always introduce small perturbations into the functions $m(r)$ and $\phi(r)$. This other (neglected) type are called polar perturbations; for this kind, a reversal of $\varphi$ does not affect the metric. In the case of a vacuum, axial and polar perturbations must decouple and any arbitrary perturbation can be written as a linear superposition. Let us note, however, that even for the simple model of a Schwarzschild black hole in a vacuum, the scattering potential for polar perturbations does not generalize to the (naive) suggestion of replacing $2 m$ with $2 m\left(1-j^{2}\right)$ in equation (12) for scalars.

We now proceed by demanding that the perturbations do not change the stress-energy tensor (at least) to first order in $q$. The non-vanishing first-order components of the Ricci tensor are found to be

$$
\delta R_{13}=\frac{1}{r^{3} \sqrt{1-2 m / r} \sin ^{2} \theta \mathrm{e}^{-\phi}}\left[q_{[r, \theta]} r^{2} \sin ^{3} \theta\left(1-\frac{2 m}{r}\right) \mathrm{e}^{-\phi}\right]_{, \theta}+\frac{r \sin \theta}{\mathrm{e}^{-2 \phi} \sqrt{1-2 m / r}} q_{[t, r], t},
$$


$\delta R_{23}=-\left(r^{2} \sin ^{2} \theta \mathrm{e}^{-\phi}\right)^{-1}\left[q_{[r, \theta]} r^{2} \sin ^{3} \theta\left(1-\frac{2 m}{r}\right) \mathrm{e}^{-\phi}\right]_{, r}+\frac{\sin \theta}{\mathrm{e}^{-2 \phi}(1-2 m / r)} q_{[t, \theta], t}$,

$\delta R_{03}=\frac{1}{r^{3} \sin ^{2} \theta}\left[\sqrt{1-2 m / r}\left(q_{[t, r]} \frac{r^{4} \sin ^{3} \theta}{\mathrm{e}^{-\phi}}\right)_{, r}+\frac{1}{r \mathrm{e}^{-\phi} \sqrt{1-2 m / r}}\left(q_{[t, \theta]} r^{3} \sin ^{3} \theta\right)_{, \theta}\right]$,

which yield the relations

$$
\begin{aligned}
& \delta R_{13}=0 \quad \Leftrightarrow \quad\left[q_{[r, \theta]} r^{2} \sin ^{3} \theta\left(1-\frac{2 m}{r}\right) \mathrm{e}^{-\phi}\right]_{, \theta}=-\frac{r^{4} \sin ^{3} \theta}{\mathrm{e}^{-\phi}} q_{[t, r], t}, \\
& \delta R_{23}=0 \quad \Leftrightarrow \quad\left[q_{[t, \theta]} r^{2} \sin ^{3} \theta\left(1-\frac{2 m}{r}\right) \mathrm{e}^{-\phi}\right]_{, r}=\frac{r^{2} \sin ^{3} \theta}{\mathrm{e}^{-\phi}(1-2 m / r)} q_{[t, \theta], t},
\end{aligned}
$$

with the third possible expression being redundant. These equations can be most easily found by tetrad methods or, alternatively, consult section 24 of Chandrasekhar [33] (equations (11) and (12)). Note that these formulae even hold true in the more general case of $g_{t t}, g_{r r}$ and $g_{\theta \theta}$ depending on $\theta$ as well.

To help simplify the above results, let us define the variable $Q$ by

$$
Q(r, \theta, t) \equiv r^{2} \sin ^{3} \theta\left[1-\frac{2 m(r)}{r}\right] \mathrm{e}^{-\phi(r)} q_{[r, \theta]} .
$$

The equations governing the perturbations ((A.5) and (A.6)) can now be expressed in the following form:

$$
\begin{aligned}
Q_{, r} & =\frac{r^{2} \sin ^{3} \theta}{\mathrm{e}^{-\phi}(1-2 m / r)} q_{[t, \theta], t}, \\
Q_{, \theta} & =-r^{4} \sin ^{3} \theta \mathrm{e}^{\phi} q_{[t, r], t} .
\end{aligned}
$$

Next, we can solve for separable harmonic perturbations by imposing a suitable ansatz,

$$
Q(r, \theta, t)=r R(r) \vartheta(\theta) \mathrm{e}^{i k t},
$$

with other solutions obtainable by superposition. Also applying the commutativity property of the partial derivatives, we can eliminate the function $q_{t}$ from equations (A.8) and (A.9) to obtain

$$
\begin{array}{r}
\vartheta\left[\frac{2 \mathrm{e}^{-\phi}(1-2 m / r)}{r^{2} \sin ^{3} \theta}(r R)_{, r}\right]_{, r}+\frac{R \mathrm{e}^{-\phi}}{r^{3}}\left[\frac{2(\vartheta)_{, \theta}}{\sin ^{3} \theta}\right]_{, \theta} \\
=k^{2}\left(q_{\theta, r}-q_{r, \theta}\right) \mathrm{e}^{-\mathrm{i} k t}=-\frac{2 k^{2} R \vartheta}{r \sin ^{3} \theta(1-2 m / r) \mathrm{e}^{-\phi}},
\end{array}
$$

which implies

$$
\frac{r^{3} \mathrm{e}^{\phi}}{R}\left[\frac{\mathrm{e}^{-\phi}(1-2 m / r)}{r^{2}}(r R)_{, r}\right]_{, r}+\frac{\sin ^{3}(\theta)}{\vartheta}\left[\frac{1}{\sin ^{3}(\theta)}(\vartheta)_{, \theta}\right]_{, \theta}+r^{2} \mathrm{e}^{2 \phi} \frac{k^{2}}{(1-2 m / r)}=0 .
$$


The terms are now in a form that is particularly suitable for finding separable solutions. Denoting the separation constant by $\gamma^{2}$, one finds that

$$
\begin{aligned}
& r^{3} \mathrm{e}^{\phi}\left[\frac{\mathrm{e}^{-\phi}(1-2 m / r)}{r^{2}}(r R)_{, r}\right]_{, r}+r^{2} \mathrm{e}^{2 \phi} \frac{k^{2}}{(1-2 m / r)} R-\gamma^{2} R=0, \\
& \sin ^{3} \theta\left[\frac{1}{\sin ^{3} \theta} \vartheta_{, \theta}\right]_{, \theta}+\gamma^{2} \vartheta=0 .
\end{aligned}
$$

The angular equation is quite easily solved and gives

$$
\vartheta(\theta)=\sin ^{2} \theta\left[A P_{2}^{\ell}(\cos (\theta))+B Q_{2}^{\ell}(\cos (\theta))\right],
$$

where

$$
\ell \equiv \frac{\sqrt{9+4 \gamma^{2}}-1}{2}
$$

$P_{m}^{\ell}$ and $Q_{m}^{\ell}$ are the Legendre polynomials, while $A$ and $B$ are arbitrary constants. To ensure that the solutions are real, we must restrict the values of $\ell$ to integers, and so

$$
\gamma^{2}=\ell^{2}+\ell-2=(\ell+2)(\ell-1) \quad \text { where } \ell=0,1,2, \ldots
$$

The radial equation is not so trivially solved. However, we can still transform it into a recognizable form by first taking note of equation (3) for the (generalized) tortoise coordinate. It follows that the radial equation can also be expressed as

$$
r^{3} \mathrm{e}^{\phi}\left[\frac{\mathrm{d} r}{\mathrm{~d} r_{*}} \frac{R}{r^{2}}+\frac{R_{, r_{*}}}{r}\right]_{, r}+r^{2} \mathrm{e}^{\phi} \frac{\mathrm{d} r_{*}}{\mathrm{~d} r} k^{2} R-\gamma^{2} R=0
$$

or

$$
\frac{\mathrm{d}^{2} R}{\mathrm{~d} r_{*}^{2}}+\frac{\mathrm{d} r}{\mathrm{~d} r_{*}}\left[\frac{1}{r} \frac{\mathrm{d}}{\mathrm{d} r}\left(\frac{\mathrm{d} r}{\mathrm{~d} r_{*}}\right) R-2\left(\frac{\mathrm{d} r}{\mathrm{~d} r_{*}}\right) \frac{R}{r^{2}}-\frac{\gamma^{2} R}{r^{2}} \mathrm{e}^{-\phi}\right]=-k^{2} R .
$$

The above can readily be identified as having the form of a one-dimensional scattering equation (cf, equation (11)) with a potential of

or

$$
V(r) \equiv \frac{\mathrm{d} r}{\mathrm{~d} r_{*}}\left[-\frac{1}{r} \frac{\mathrm{d}}{\mathrm{d} r}\left(\frac{\mathrm{d} r}{\mathrm{~d} r_{*}}\right)+2\left(\frac{\mathrm{d} r}{\mathrm{~d} r_{*}}\right) \frac{1}{r^{2}}+\frac{\gamma^{2}}{r^{2}} \mathrm{e}^{-\phi}\right]
$$

$$
V(r)=\mathrm{e}^{-2 \phi}\left(1-\frac{2 m}{r}\right)\left[\frac{\ell(\ell+1)}{r^{2}}-\frac{6 m}{r^{3}}+\frac{2 m_{, r}}{r^{2}}+\left(1-\frac{2 m}{r}\right) \frac{\phi_{, r}}{r}\right] .
$$

(As a consistency check, it can be verified that this form correctly reduces to the potential given in [22] for the same type of perturbation in a Schwarzschild-de Sitter background.) This equation can be somewhat simplified by noting that the two derivative terms appearing above can be rewritten in terms of the $R_{\hat{\theta} \hat{\theta}}$ (or $R_{\hat{\varphi} \hat{\varphi}}$ ) orthonormal component of the Ricci tensor:

$$
V(r)=\mathrm{e}^{-2 \phi}\left(1-\frac{2 m}{r}\right)\left[\frac{\ell(\ell+1)}{r^{2}}-\frac{6 m(r)}{r^{3}}+R_{\hat{\theta} \hat{\theta}}(r)\right] .
$$

Equivalently, in terms of the Einstein tensor

$$
V(r)=\mathrm{e}^{-2 \phi}\left(1-\frac{2 m}{r}\right)\left[\frac{\ell(\ell+1)}{r^{2}}-\frac{6 m(r)}{r^{3}}+\frac{G_{\hat{t} \hat{t}}-G_{\hat{r} \hat{r}}}{2}\right] .
$$

If desired, the Einstein equations could be used to rewrite the trailing term in terms of the density and radial pressure. Comparing this potential with equation (13) for a scalar perturbation, one can see that there is no obvious generalization when the black hole is truly 'dirty'. As a 
further point of interest, one can also see (most easily from equation (A.20)) that, in the limit of squeezed horizons, only the $\gamma^{2}$ term will contribute to the scattering.

As a second consistency check this can be compared with the analysis of Karlovini [32], to which it is equivalent for $j=2$. In fact Karlovini also performed the $j=1$ calculation for the Maxwell field. Combined with the (minimally coupled) scalar result as discussed earlier in this paper it is possible to recast Karlovini's result in the form

$V(r)=\mathrm{e}^{-2 \phi}\left(1-\frac{2 m}{r}\right)\left[\frac{\ell(\ell+1)}{r^{2}}+\left(1-j^{2}\right) \frac{2 m(r)}{r^{3}}-(1-j) R_{\hat{\theta} \hat{\theta}}\right]$,

for $j=0,1,2$. Given the results of the scalar, Maxwell and gravity computations, deriving this is a curve-fitting exercise on a curve with three points. There is therefore no particular reason to trust this formula for higher values of $j$.

\section{References}

[1] Press W H 1971 Long wave trains of gravitational waves from a vibrating black hole Astrophys. J. 170 L105

[2] Bachelot A and Motet-Bachelot A Resonances of Schwarzschild black holes Proc. IV Int. Conf. of Hyperbolic Problems (Braunschweig: Vieweg) (Taosmina, 1992)

[3] See, for comprehensive reviews Nollert H-P 1999 Quasinormal modes: the characteristic sound of black holes and neutron stars Class. Quantum Grav. 16 R159

Kokkotas K D and Schmidt B G 1999 Quasi-normal modes of black holes and stars Living Rev. Rel. 22 (Preprint gr-qc/9909058)

[4] Hod S 1998 Bohr's correspondence principle and the area spectrum of quantum black holes Phys. Rev. Lett. 81 4293 (Preprint gr-qc/9812002)

[5] Bekenstein J D 1974 The quantum mass spectrum of the Kerr black hole Lett. Nuovo Cimento 11467

[6] Nollert H-P 1993 Quasinormal modes of Schwarzschild black holes: the determination of quasinormal frequencies with very large imaginary parts Phys. Rev. D 475253

[7] Andersson N 1993 On the asymptotic distribution of quasinormal-mode frequencies for Schwarzschild black holes Class. Quantum Grav. 10 L61

[8] Dreyer O 2003 Quasinormal modes, the area spectrum, and black hole entropy Phys. Rev. Lett. 90081301 (Preprint gr-qc/0211076)

[9] Kunstatter G $2003 d$-dimensional black hole entropy spectrum from quasi-normal modes Phys. Rev. Lett. 90 161301 (Preprint gr-qc/0212014)

[10] Berti E and Kokkotas K D 2003 Asymptotic quasinormal modes of Reissner-Nordstrom and Kerr black holes Phys. Rev. D 68044027 (Preprint hep-th/0303029)

[11] Glampedakis K and Andersson N 2003 Quick and dirty methods for studying black-hole resonances Class. Quantum Grav. 203441 (Preprint gr-qc/0304030)

[12] Motl L 2003 An analytical computation of asymptotic Schwarzschild quasinormal frequencies Adv. Theor. Math. Phys. 61135 (Preprint gr-qc/0212096)

[13] Motl L and Neitzke A 2003 Asymptotic black hole quasinormal frequencies Adv. Theor. Math. Phys. 7307 (Preprint hep-th/0301173)

[14] Neitzke A 2003 Greybody factors at large imaginary frequencies Preprint hep-th/0304080

[15] Medved A J M, Martin D and Visser M 2003 Dirty black holes: quasinormal modes Preprint gr-qc/0310009

[16] Cardoso V, Lemos J P and Yoshida S 2003 Quasinormal modes of Schwarzschild black holes in four and higher dimensions Preprint gr-qc/0309112

[17] Visser M 1992 Dirty black holes: thermodynamics and horizon structure Phys. Rev. D 462445 (Preprint hep-th/9203057)

[18] Visser M 1993 Dirty black holes: Entropy versus area Phys. Rev. D 48583 (Preprint hep-th/9303029)

[19] Visser M 1993 Dirty black holes: Entropy as a surface term Phys. Rev. D 485697 (Preprint hep-th/9307194)

[20] Padmanabhan T 2003 Quasi normal modes: a simple derivation of the level spacing of the frequencies Preprint gr-qc/0310027

[21] Poschl G and Teller E 1933 Z. Phys. 83143

[22] Cardoso V and Lemos J P S 2003 Quasinormal modes of the near extremal Schwarzschild-de Sitter black hole Phys. Rev. D 67084020 (Preprint gr-qc/0301078)

[23] Visser M 1995 Lorentzian Wormholes: From Einstein To Hawking (Woodbury: AIP Press) 
[24] Morris M S and Thorne K S 1988 Wormholes in space-time and their use for interstellar travel: a tool for teaching general relativity Am. J. Phys. 56395

[25] Gibbons G W and Hawking S W 1977 Cosmological event horizons, thermodynamics and particle creation Phys. Rev. D 152738

[26] Nariai H 1950 Sci. Rep. Tohuku Univ. 34160

[27] Regge T and Wheeler J A 1957 Stability of a Schwarzschild singularity Phys. Rev. 1081063

[28] Zerilli F J 1970 Gravitational field of a particle falling in a Schwarzschild geometry analyzed in tensor harmonics Phys. Rev. D 22141

[29] Suneeta V 2003 Quasinormal modes for the SdS black hole: an analytical approximation scheme Phys. Rev. D 68024020 (Preprint gr-qc/0303114)

[30] Ferrari V and Mashhoon B 1984 Phys. Rev. D 30295

[31] For general spins, see Kanti P and March-Russell J 2003 Calculable corrections to brane black hole decay: II. Greybody factors for spin 1/2 and 1 Phys. Rev. D 67104019 (Preprint hep-ph/0212199)

Cvetič M and Larsen F 1998 Greybody factors for black holes in four dimensions: particles with spin Phys. Rev. D 576297 (Preprint hep-th/9712118)

[32] Karlovini M 2002 Axial perturbations of general spherically symmetric spacetimes Class. Quantum Grav. 19 2125 (Preprint gr-qc/0111066)

[33] Chandrasekhar S 1983 The Mathematical Theory of Black Holes (Oxford: Science Publications) 